\title{
Weißer Hautkrebs - jedes Jahr mehr Neuerkrankungen
}

\begin{abstract}
Weltweit steigt die Zahl der nicht melanozytären Hautkrebserkrankungen von Jahr zu Jahr. Das ist in Deutschland nicht anders, wie Dermatologen aus Tübingen hochgerechnet haben. Und eine Trendwende ist nicht in Sicht.
\end{abstract}

Um einen Eindruck zu bekommen, wie sich in Deutschland über die Jahre die Erkrankungszahlen nicht melanozytärer Karzinome der Haut (NMSC) entwickelt haben, warfen die Dermatologin Ulrike Leitner von der Universität in Tübingen und Kollegen einen Blick auf die Krebsregister im Saarland und in Schleswig-Holstein.

Laut Krebsregister des Bundeslandes Schleswig-Holstein ist zwischen den Jahren 1999 und 2012 die Rate an jährlichen Neuerkrankungen stark gestiegen, von 147 auf 278 Fälle pro 100.000 Personenjahre bei Männern und von 143 auf 241 Fälle pro 100.000 Personenjahre bei Frauen. Das entspricht einem durchschnittlichen jährlichen Zuwachs von 5,0\% (95\%-KI 4,4-5,7) bzw. 4,4\% (95\%-KI 3,6-5,1). Nicht ganz so steil fiel der Anstieg der altersstandardisierten Inzidenzraten aus: Sie sind bei Männern von 125 auf 170 Fälle pro 100.000 Personenjahre geklettert, bei Frauen von 92 auf 134.

Im Saarland zeichnete sich eine noch deutlichere Entwicklung ab: Die Inzidenzraten erhöhten sich in den Zeiträumen 1970-1972 und 2010-2012 bei Männern von 8,4 auf 186,1 Fälle pro 100.000 Personenjahre, was einem durchschnittlichen jährlichen Anstieg von 7,7\% (95\%-KI 6,4-9,0) entspricht, und bei Frauen von 9,1 auf 163,1 Fälle pro 100.000 Personenjahre (average annual percentage of change $[\mathrm{AAPC}]=+7,4 \%$; $95 \%$-KI $6,4-8,5)$. Wie auch in Schleswig-Holstein blieben die altersadjustierten Inzidenzraten etwas darunter und stiegen bei Männern von 10,5 auf 117,7 Fälle pro 100.000 Personenjahre, bei Frauen von 8,1 auf 93,1.

\section{Sterberaten folgen einem anderen Trend}

Erfreulicher stimmt der Blick auf die Mortalitätsraten. Der Anteil der Patienten, die an ihrem Basalzell- oder Plattenepithelkarzinom gestorben sind, blieb im zeitlichen Verlauf entweder stabil oder ist sogar gesunken. Für das Saarland ermittelten die Dermatologen für den Zeitraum von 1970-1972 eine altersstandardisierte, auf 100.000 Personenjahre bezogene Mortalitätsrate von 0,96 bei Männer bzw. 0,86 für Frauen, die später (2003-2012) bei beiden Geschlechtern auf 0,8 gesunken ist. In Schleswig-Holstein wiederum hat sich die Rate bei Männern von 0,51 (1999-2001) auf 0,46 (2010-2012) verringert, während sie bei Frauen weitgehend stabil geblieben ist $(0,16$ vs. 0,19$)$.

\section{Keine Trendwende vor 2050}

In Deutschland seien innerhalb der letzten 43 Jahre die Erkrankungszahlen im Hinblick auf den weißen Hautkrebs kontinuierlich gestiegen, so das Resümee der Tübinger Dermatologen. Neben der Einführung des Hautkrebsscreenings habe sicherlich auch die Verbesserung des NMSC-Registrierungsprozesses zu dieser Entwicklung beigetragen, wie die Studienautoren erklären. Das Hautkrebsscreening sowie das größere Bewusstsein in der Bevölkerung für nicht melanozytäre Hautkrebserkrankungen habe außerdem zu einer früheren Diagnose und damit besseren Therapieaussichten geführt, was zu den sinkenden bzw. stabilen Sterberaten beigetragen hat.

Ein Ende der steigenden Erkrankungszahlen sei in naher Zukunft leider nicht in Sicht, davon sind die Studienautoren überzeugt. Vielmehr erwarten sie mit Blick auf die demografische Entwicklung und die steigende UV-Belastung eine Verdopplung der Inzidenzrate innerhalb der nächsten 15 Jahre. Vor 2050 ist mit einer Trendwende nach Ansicht der Tübinger Dermatologen nicht zu rechnen.

Dr. Dagmar Kraus

Literatur

1. Leiter U et al. J Invest Dermatol 2017;137:1860-7

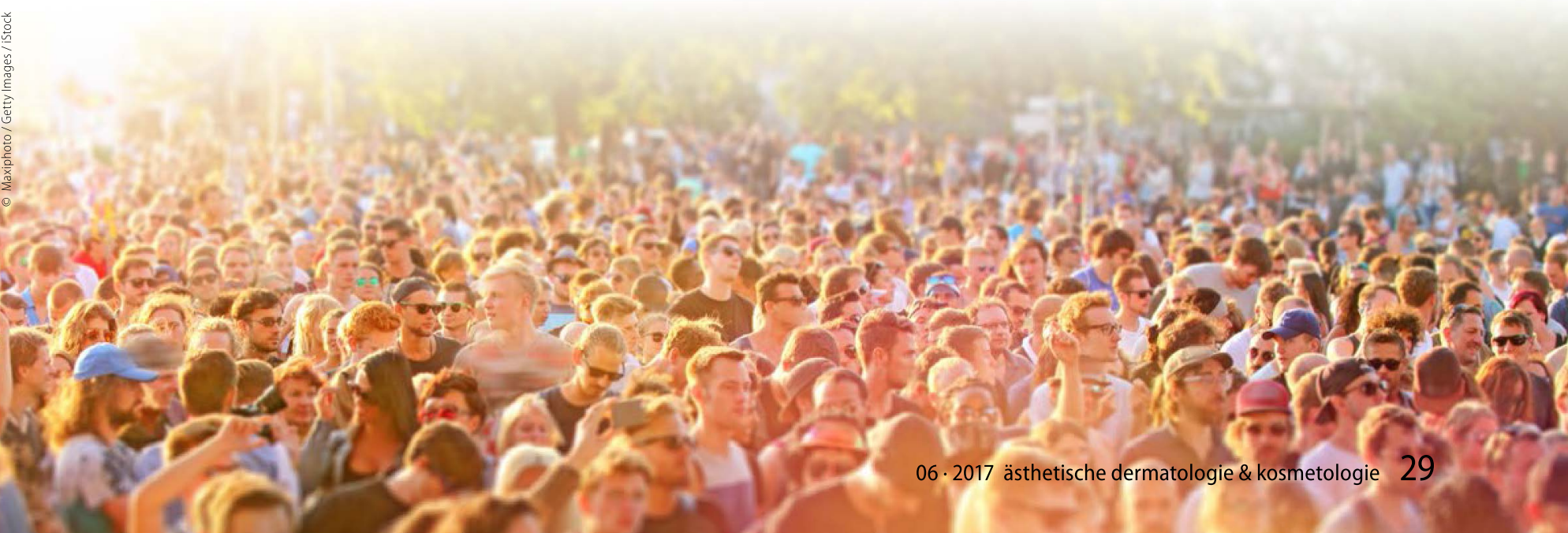

